\section{JTI}

JOURNAL OF

TRAUMA AND INJURY

\title{
Iatrogenic Duodenal Obstruction due to Acupuncture Therapy Trauma
}

\author{
Jae Hun Chung, M.D. ${ }^{1}$, Si-Hak Lee, M.D., Ph.D. ${ }^{1,2}$ \\ ${ }^{1}$ Department of Surgery, Pusan National University Yangsan Hospital, Yangsan, Korea \\ ${ }^{2}$ Research Institute for Convergence of Biomedical Science and Technology, Pusan National \\ University Yangsan Hospital, Yangsan, Korea
}

Received: May 28, 2020

Revised: August 4, 2020

Accepted: August 26, 2020

\section{Correspondence to}

Si-Hak Lee, M.D., Ph.D.

Division of Gastrointestinal, Department of Surgery, Pusan National University

Yangsan Hospital, 20 Geumo-ro, Moolgeum-eup, Yangsan 50612, Korea

Tel: +82-55-360-2124

Fax: +82-55-360-2154

E-mail: ghost109@pusan.ac.kr
There are many possible causes of duodenal obstruction, such as congenital anomalies and various acquired conditions associated with space-occupying lesions. However, hemorrhage or retroperitoneal hematoma is a rare cause of duodenal obstruction. Here, we report the case of a 55-year-old man who developed duodenal obstruction due to a large retroperitoneal hematoma after acupuncture therapy. The patient experienced abdominal discomfort along with vomiting and nausea. Considering the size of the hematoma, emergency surgery could have been performed, but conservative treatment was continued because the patient's vital signs were stable. With spontaneous resolution of the hematoma, the symptoms of duodenal obstruction improved. The patient was eventually discharged without any complications associated with the hematoma. Our findings suggest that even when a hematoma is large, a conservative approach can be maintained until improvement of the symptoms of duodenal obstruction if the vital signs of the patient remain stable.

Keywords: Acupuncture; Duodenal obstruction; Hematoma

\section{INTRODUCTION}

There are many possible causes of duodenal obstruction, such as congenital anomalies and various acquired conditions associated with space-occupying lesions [1]. The specific retroperitoneal position of the duodenum predisposes it to compression, displacement, and obstruction from a myriad of retroperitoneal disorders. The list includes inflammatory or infective etiologies, cystic or solid masses of genitourinary origin, hemorrhage, and primary retroperitoneal tumors [2]. Lymphoma, which is a common retroperitoneal tumor, can cause compression of the duodenal lumen, but 
strictures are rare. Primary retroperitoneal tumors, such as liposarcoma (40\%), leiomyosarcoma (30\%), and malignant fibrous histiocytoma (15\%), are known to reach large sizes and can cause duodenal obstruction. Hemorrhage or a retroperitoneal hematoma is a rare cause of duodenal obstruction compared with tumor compression [3]. Idiopathic retroperitoneal hematoma is a rare condition, and few cases have been reported in the literature, although it was first described by Barber in 1909 [4]. Here, we report a case of duodenal obstruction due to an idiopathic retroperitoneal hematoma associated with acupuncture therapy.

\section{CASE REPORT}

A 55-year-old man was transferred to the emergency room (ER) because of abdominal discomfort along with vomiting and nausea. He had no specific medical history, such as anticoagulation medication management. However, he had experienced back pain for a long time, and he underwent acupuncture therapy for pain control. His back pain had been treated with acupuncture therapy a week before the appearance of his symptoms, including abdominal discomfort, vomiting, and nausea. He stated that he had been treated with long-needle acupuncture therapy, and that this was the only difference from his usual acupuncture therapy.

Laboratory studies revealed hyperbilirubinemia (serum total bilirubin level, $2.2 \mathrm{mg} / \mathrm{dL}$ ) and an elevated high-sensitivity C-reactive protein (hsCRP) level $(8.68 \mathrm{mg} / \mathrm{dL}$ ). Considering his history and presentation, the staff at the ER performed contrast-enhanced computed tomography (CT) and requested a surgical consultation. The CT scan revealed distention of the stomach (Fig. 1A) and high-density fluid in the right anterior pararenal space, suggesting a hematoma that was compressing the second portion of the duodenum (Fig. 1B). Additionally, initial erect supine radiography of the abdomen showed numerous needle tips, which were associated with his previous treatment for back pain (Fig. 2). We made a diagnosis of gastric outlet obstruction, probably associated with an iatrogenic retroperitoneal hematoma caused by the long-needle acupuncture therapy performed a week before the patient visited the ER.

As his vital signs were stable and his hemoglobin level
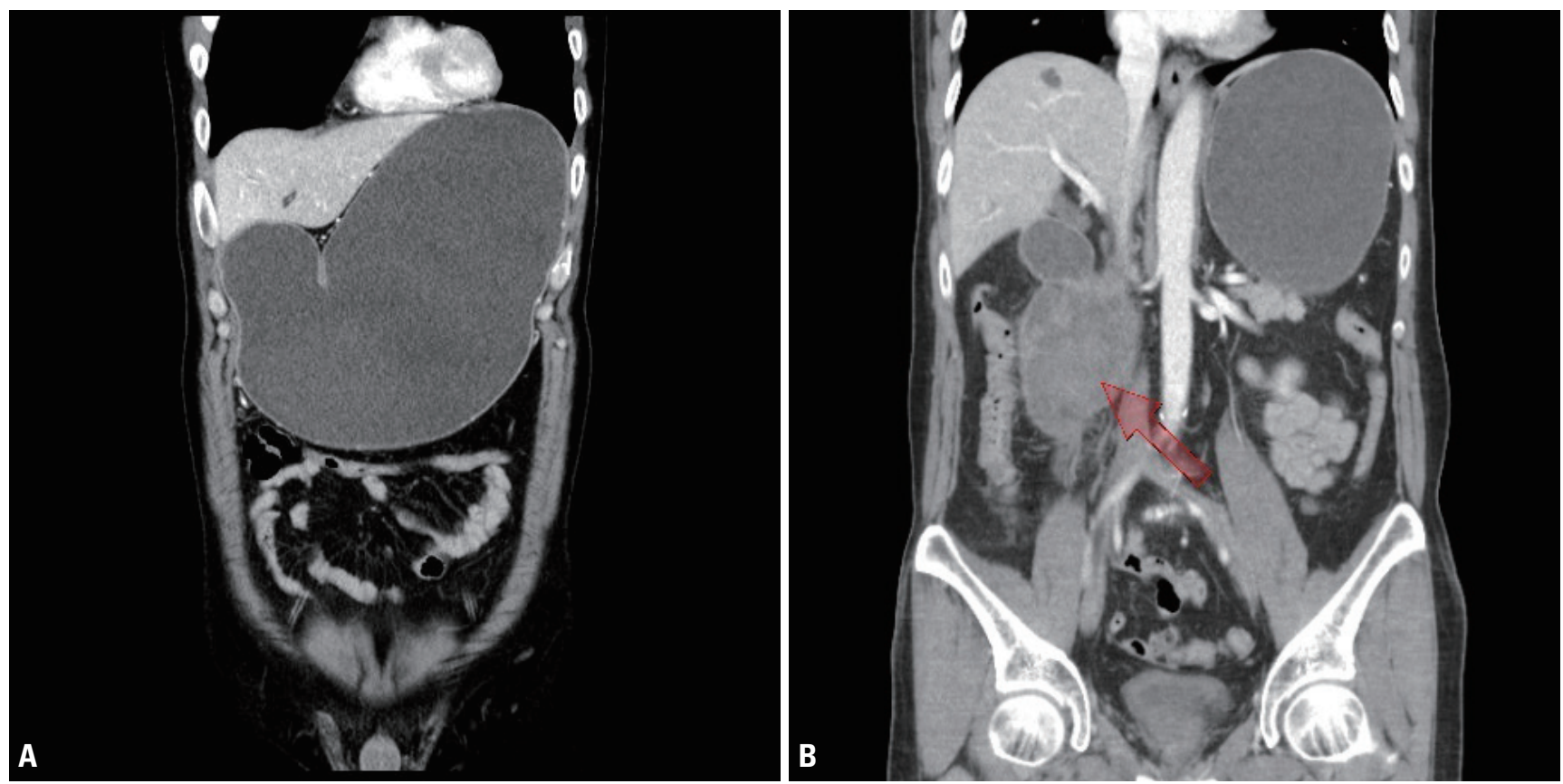

Fig. 1. Initial computed tomography (CT) scan in the emergency room. (A) The initial CT scan revealed distention of the stomach. (B) High-density fluid in the right anterior pararenal space, suggestive of hematoma (arrow, $9.6 \mathrm{~cm}$ ), caused narrowing of the second portion of the duodenum. 


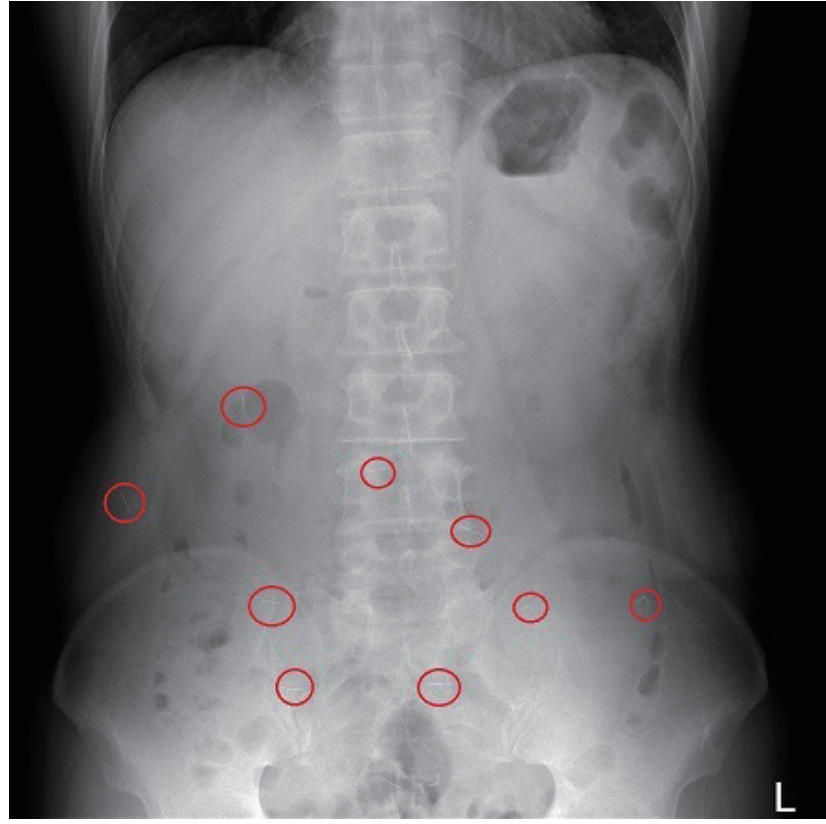

Fig. 2. Initial erect supine radiography of the abdomen in the emergency room showed numerous needle tips (red circles), which were associated with his previous acupuncture therapy for back pain.
$(13.3 \mathrm{~g} / \mathrm{dL})$ was almost in the reference range (13.5-17.5 $\mathrm{g} / \mathrm{dL}$ ), we planned to treat him in a conservative manner with Levin tube insertion and prophylactic antibiotics for inflammatory changes and ongoing infection associated with the hematoma. The administered antibiotics involved a combination of a third-generation cephalosporin (ceftriaxone) and metronidazole. A Levin tube was inserted to reduce gastric distension. As the volume of tube drainage decreased, his symptoms associated with the gastric outlet obstruction improved. Daily erect supine radiography of the abdomen was performed, and we confirmed improvements in the radiography findings. Additionally, the hsCRP level and serum white blood cell count decreased from $8.68 \mathrm{mg} / \mathrm{dL}$ and $14.27 \times 10^{3}$ cells $/ \mu \mathrm{L}$, respectively, to $2.35 \mathrm{mg} / \mathrm{dL}$ and $6.63 \times 10^{3}$ cells $/ \mu \mathrm{L}$, respectively, on postadmission day 3 .

The first follow-up CT scan was performed 10 days after starting supportive care. The CT scan revealed a decrease in the size of the retroperitoneal hematoma from $9.6 \mathrm{~cm}$ to $7.1 \mathrm{~cm}$, and there was no evidence of dilatation of the
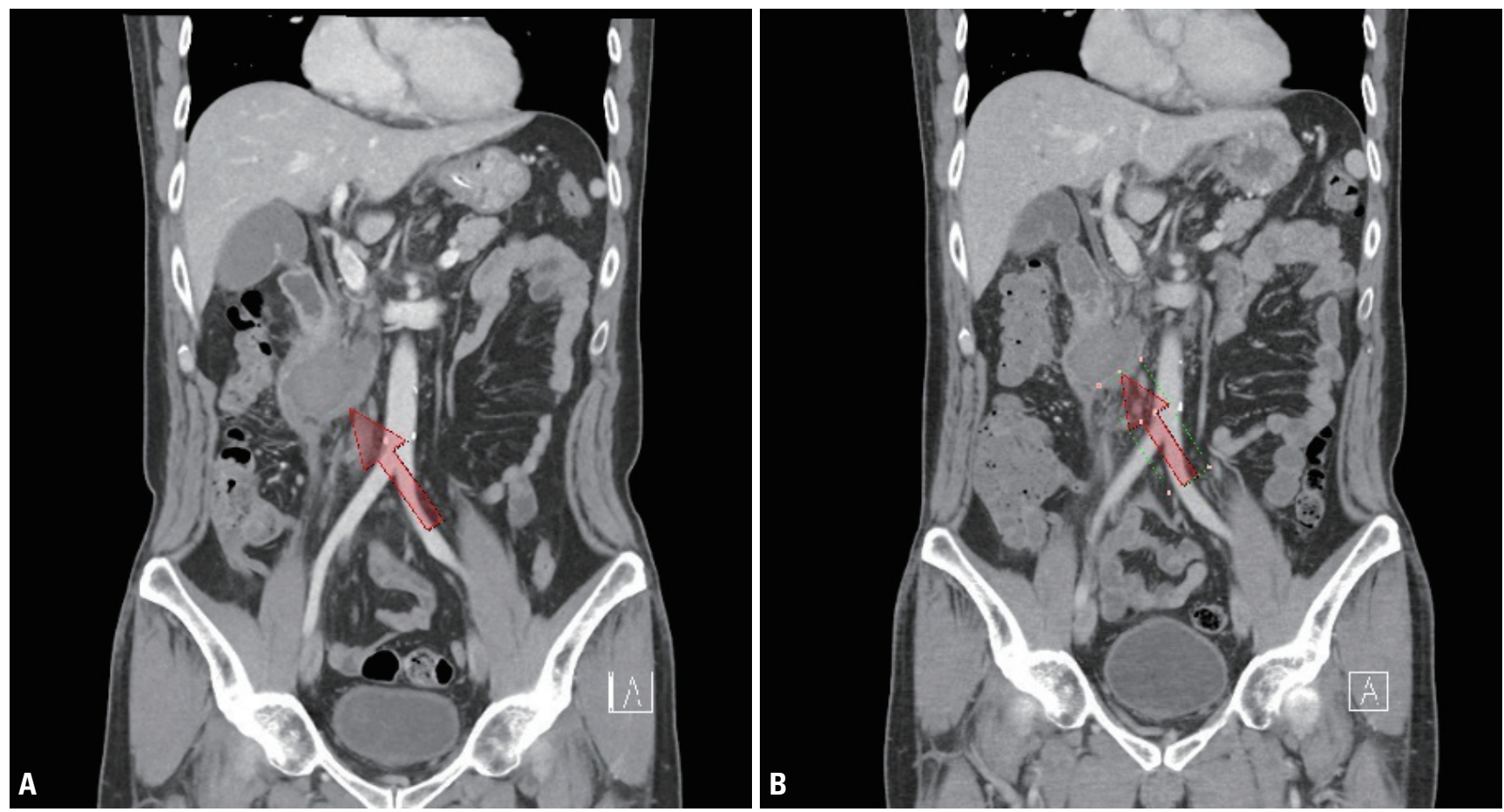

Fig. 3. Follow-up computed tomography (CT) on admission days 10 and 16. (A) CT revealed a decrease in the volume of high-density fluid in the right anterior pararenal space, suggestive of hematoma (arrow, 9.6 $\rightarrow 7.1 \mathrm{~cm}$ ), on admission day 10. (B) CT revealed a decrease in the volume of high-density fluid in the right anterior pararenal space, suggestive of hematoma (arrow, $7.1 \rightarrow 5.4 \mathrm{~cm}$ ), on admission day 16. Finally, there is no evidence of dilatation of the stomach and duodenal bulb. 
stomach and duodenal bulb (Fig. 3A). As the hematoma resolved, we found that the mass effect causing duodenal obstruction improved. The second follow-up CT scan also revealed a decrease in the amount of high-density fluid in the right anterior pararenal space, suggesting a decrease in the size of the hematoma from $7.1 \mathrm{~cm}$ to $5.4 \mathrm{~cm}$ (Fig. 3B).

During the nil per os period, nutritional support was provided by total parenteral nutrition (SmofKabiven ${ }^{\circledR}$, Fresenius Kabi Korea Ltd, Seoul, Korea) via a peripherally inserted central catheter. Smofkabiven ${ }^{\circledast}$ (Fresenius Kabi Korea Ltd) is a 3-chamber bag with separate compartments providing a combination of amino acids, lipids, and glucose. It contains a next-generation lipid emulsion with fish oil, medium chain triglycerides, olive oil, and soybean oil. After confirmation of a decrease in the size of the retroperitoneal hematoma, an oral diet was started on postadmission day 13 . The oral diet was gradually advanced from a clear liquid diet to a soft diet. During this gradual change in the diet, the patient had no gastrointestinal symptoms, such as dyspepsia and abdominal pain. He was eventually discharged on postadmission day 17 , without any sequelae or complications.

A week after discharge, the patient visited the outpatient department for follow-up and did not have any sequelae or complications. His diet was well-tolerated, and he reported no longer experiencing dyspepsia and abdominal pain. He was told to visit the ER if symptoms, such as vomiting, fever, and abdominal pain, worsened. If no specific symptoms occur, outpatient follow-up is scheduled for CT and assessment of hsCRP levels after 6 months.

\section{DISCUSSION}

An iatrogenic retroperitoneal hematoma is a rare condition. The clinical symptoms depend on the size and location of the hematoma. Ongoing active hemorrhage of a retroperitoneal hematoma can cause intra-abdominal hemoperitoneum, which can be fatal; thus, surgical intervention should be considered. Several well-known common causes of retroperitoneal hemorrhage are abdominal aortic or visceral artery aneurysms, coagulopathy, and retroperitoneal neoplasms [5]. Therefore, management of a retroperitoneal hematoma should depend on the patient's vital signs, the cause of hemorrhage, and the location of the hematoma. If there is an accompanying adjacent organ injury that requires surgical intervention, surgery should be considered to repair the injury, even when vital signs are stable.

Acupuncture has been used in the treatment of pain and other medical ailments for centuries, and its usage is reportedly increasing globally. Although the frequency of reported acupuncture-associated complications appears to be steady over time, rare adverse effects and complications should arouse concerns [6]. Among the complications associated with acupuncture therapy, a retroperitoneal hematoma is a relatively rare finding, and mainly occurs due to vascular accidents [7] or excessive anticoagulation [8].

In our case, the patient's symptoms upon presentation were probably due to duodenal compression associated with a large hematoma that caused substenosis, rigidity, and fixation of the lumen, leading to gastric stasis and vomiting. As the patient's vital signs were stable and the hemoglobin level was in the reference range, we planned to treat the patient in a conservative manner. In the possible event of emergency surgery, we prepared for abdominal compartment syndrome due to rebleeding or aggravation of mechanical ileus. However, the spontaneous resolution of the hematoma improved the duodenal obstruction symptoms, and the patient did not require operative management.

According to the Bologna guidelines for the diagnosis and management of adhesive small bowel obstruction (ASBO) [9], upper gastrointestinal (GI) or small bowel series with water-soluble contrast medium can be a non-operative treatment for ASBO. However, in our case, the patient had an obvious history and a CT image showing obstructive symptoms, which were associated with the mass effect of the hematoma and not an adhesive lesion. Thus, we decided not to perform upper GI or small bowel series as a non-operative treatment, but we considered upper GI or small bowel series to evaluate bowel passage. However, during the admission period, symptom relief was very dramatic and obvious with a sip of water. Thereafter, the patient was started on a clear liquid diet, and there were no symptoms of nausea, vomiting, dyspepsia, and abdominal pain. As the improvement of obstructive 
symptoms was definitive, we decided to skip upper GI or small bowel series.

In conclusion, we report a case of reversible duodenal obstruction due to a large retroperitoneal hematoma after acupuncture therapy. We investigated the history of the patient and determined that acupuncture therapy can cause severe bleeding and that a space-occupying large hematoma can lead to mechanical obstruction of the duodenum. Our findings suggest that even when a hematoma is large, conservative approach can be maintained until improvement of the symptoms of duodenal obstruction if the vital signs of the patient remain stable.

In addition, close observation after acupuncture is recommended for both licensed oriental medicine practitioners and unlicensed acupuncture therapists. Moreover, they should educate patients who are treated with acupuncture to visit the ER in case of any abnormal symptoms.

\section{REFERENCES}

1. Khanna S, Gupta P, Khanna R, Dalela D. Distal duodenal obstruction: a surgical enigma. Indian J Surg 2017;79:245-53.

2. Bunyaratavej K, Trisal V, Penney DG, Silapaswan S. Gallbladder hypokinesia: an unusual cause of duodenal obstruction. Am Surg 1998;64:441-3.

3. Teoh JY, Chan NH, Cheung HY, Hou SS, Ng CF. Bleeding renal angiomyolipoma presenting as duodenal obstruction. Int Urol Nephrol 2013;45:975-7.

4. Nair HT, Muscroft TJ. Idiopathic retroperitoneal haematoma. Br J Clin Pract 1992;46:148.

5. Al-Khulaiwi A, Razaak FA, El Shair A, Bamehriz F. Idiopathic retroperitoneal hematoma. Ann Saudi Med 2000;20:270-1.

6. Wu J, Hu Y, Zhu Y, Yin P, Litscher G, Xu S. Systematic review of adverse effects: a further step towards modernization of acupuncture in China. Evid Based Complement Alternat Med 2015;2015:432467.

7. Kabaroudis A, Papaziogas B, Papaziogas T. Spontaneous retroperitoneal hematoma caused by aneurysm of the inferior pancreaticoduodenal artery. Am J Surg 2002;184:174-5.

8. Mrug M, Mishra PV, Lusane HC, Cunningham JM, Alpert MA. Hemothorax and retroperitoneal hematoma after anticoagulation with enoxaparin. South Med J 2002;95:936-8.

9. Ten Broek RPG, Krielen P, Di Saverio S, Coccolini F, Biffl WL, Ansaloni L, et al. Bologna guidelines for diagnosis and management of adhesive small bowel obstruction (ASBO): 2017 update of the evidence-based guidelines from the world society of emergency surgery ASBO working group. World J Emerg Surg 2018;13:24. 